\title{
Finite-element Analysis of Spherical BOP in Snubbing Service of Gas Well
}

\author{
Zou Longqing ${ }^{1, a}$, Yu Chongpeng ${ }^{* 1, b}$ and Shao Junli ${ }^{1, c}$ \\ ${ }^{1}$ School of Northeast Petroleum University, Daqing 163318, China; \\ a350800320@qq.com, *Corresponding author \\ Email: bidane668@163.com, 'shaojunligoddess@126.com
}

Keywords: spherical BOP, shell, rubber core, finite.

\begin{abstract}
In snubbing service, the spherical BOP plays a vital role in shut-in well and running and pulling tubing. The shell and rubber core are the crucial components of spherical BOP, taking the three-dimensional software SolidWorks as a platform, these two components 3D model have been established, and the finite element calculation on them have been carried. The calculation results show that under the load of 21MPa, The maximum Von Mises pressure appeared in the junction of ball cover and base, which is 169MPa, the strength of shell structure meets the requirements. The maximum Von Mises pressure of rubber core, which is 348MPa, it also meet the the requirements. The finite-element analysis could provide reference basis for the design of spherical BOP in snubbing service.
\end{abstract}

\section{Introduction}

The single gas well in middle-shallow formation has low oil production and producing pressure, normal well killing job will cause a serious damage to the reservoir, and the capacity of parts of gas well decreased dramatically after servicing.The technology of snubbing service should be use in order to stabilize output,and the reservoir of natural gas well has strong sensitivity,that urgently required technology of snubbing service to conduct well completion and repairing.Snubbing service is an advanced well repairing technology, which accomplishes wellhead seal, conducts running and pulling tubing and well stimulation by snubbing serviced equipment controlling the pressure of wellhead under the condition that the wellhead has pressure. The snubbing service on producing well can effectively solve the contaminated problem in oil layer caused by kill fluid, it could not only protect the oil layer but also increase the output of oil and gas well[1].

Snubbing service need spherical BOP to control the pressure of tubing-casing annulus, the spherical BOP is the crucial component of insulating high temperature and high pressure in well when snubbing servicing. It could control the wellhead seal by the opening and closing of the rubber core of spherical BOP, thus accomplishing a series of snubbing service such running and pulling tubing[2]. This paper has carried finite-element analysis on spherical BOP through Solidworks software.

\section{Working principle of spherical BOP in snubbing service}

Spherical BOP is consisted of coping 、 rubber core v piston、 wear-resistant ring shell and bolt set. During shut-in, the hydraulic oil in underside oil chamber(shut-in oil chamber) push the piston to move up quickly. When arched supporting tendons of slip spherical BOP from top to bottom along the spherical inner surface of coping, the rubber between supporting tendons are forced to extruding 、 folding and deforming from edge to center, thus achieving shut-in. During well opened, the hydraulic oil in upside oil chamber(well opened oil chamber) push the piston to move down, the extrusion pressure that rubber core suffered disappeared, rubber core resuming original shape and then wellhead was opened.

The BOP group adopt two spherical BOP. In the process of running or pulling tubing, rubber core is in closed state. When tubing coupling passing through, to avoid damaging rubber core, the 
rubber core of spherical BOP need open. When the rubber core of one BOP opened, another one is closed. The stations that opening or closing of these two rubber core are alternating, thus sealing the annulus of tubing and casing[3].

\section{The modeling and finite-element analysis of spherical BOP}

When carrying finite-element analysis on spherical BOP, certain assumptions were made, as follows:

1.All materials are homogeneous and isotropic;

2.The stress and strain of shell and rubber core of spherical BOP meet Hooke's law.

On the basis of above assumptions, simplifying the force as following:

1.The pressure between shell and rubber core are negligible;

2.The maximum tear force between rubber of rubber core and framework is small, and the direction is difficult to determine, so tear force is negligible[4].

\section{The modeling and finite-element analysis of shell}

Taking the FH18-35 type spherical BOP as an example, the shell is consisted of ball cover and base. According to the actual size, using Solidworks software to set up the model of shell, as shown in Figure 1.The model is divided into grid by the method of free partition, thus generating finite element model, as shown in Figure 2. The number of nodes of divided finite element model is 25735, the number of element is 15092, the material parameters is shown in Table 1.

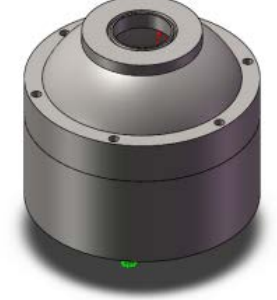

Fig. 1 The model of shell

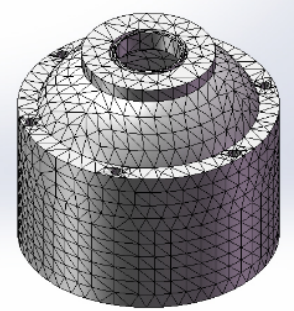

Fig. 2 The finite-element model of shell Table 1 material parameters of shell

\begin{tabular}{cc}
\hline Parameter term & Ordinary steel \\
\hline Elastic modulus/MPa & 210000 \\
Poisson ratio & 0.28 \\
Yield strength/MPa & 220 \\
Tensile strength $/ \mathrm{MPa}$ & 399 \\
Mass density $/\left(\mathrm{kg} \cdot \mathrm{m}^{-3}\right)$ & 7800 \\
Shear modulus $/ \mathrm{MPa}$ & 79000 \\
\hline
\end{tabular}

Imposing constraints on the top and bottom of the model of shell, carrying uniform load of 21MPa on all area inside the shell, and then calculating.

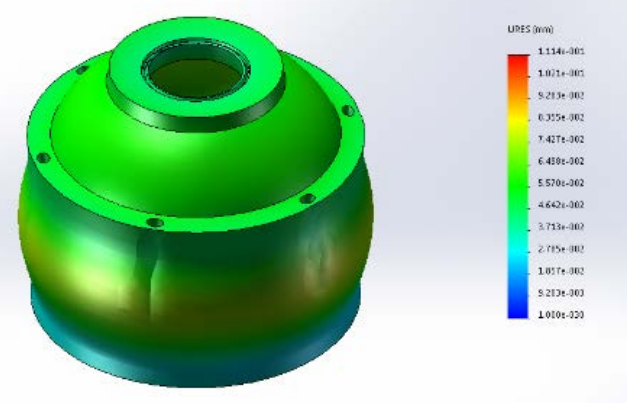

Fig. 3 The displacement nephogram of shell

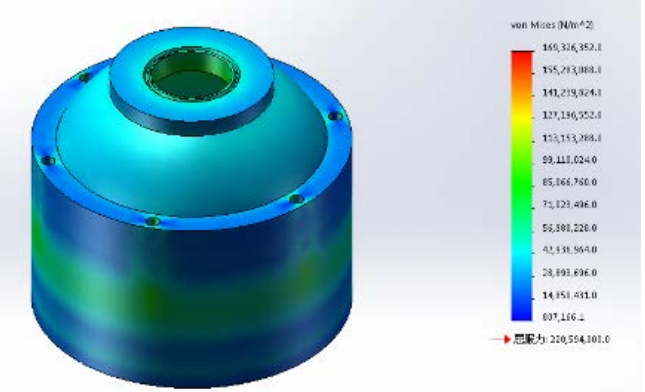

Fig.4 The stress nephogram of shell

According to the calculation result, the junction of ball cover and base is biggest, the relative displacement is $0.1114 \mathrm{~mm}$, as shown in Figure 3. The maximum Von Mises pressure also appear in 
this place,which is $169 \mathrm{MPa}$, it is less than yield strength, as shown in Figure 4 . The above shows that the shell meets the requirements.

\section{The modeling and finite-element analysis of rubber core}

According to the actual size, using Solidworks software to set up the model of rubber core, as shown in Figure 5.The model is divided into grid by the method of free partition, thus generating finite element model, as shown in Figure 6. The number of nodes of divided finite element model is 29190, the number of element is 91212, the material parameters is shown in Table 2.

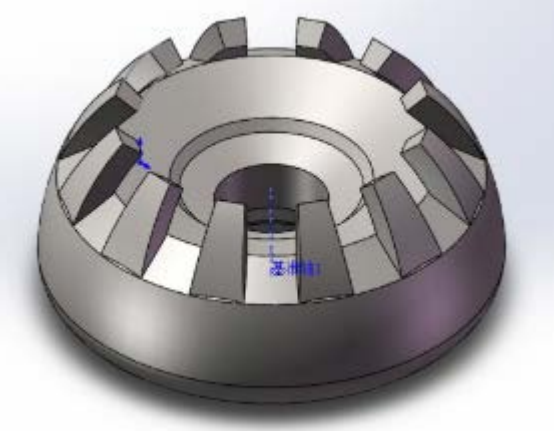

Fig. 5 The model of rubber core

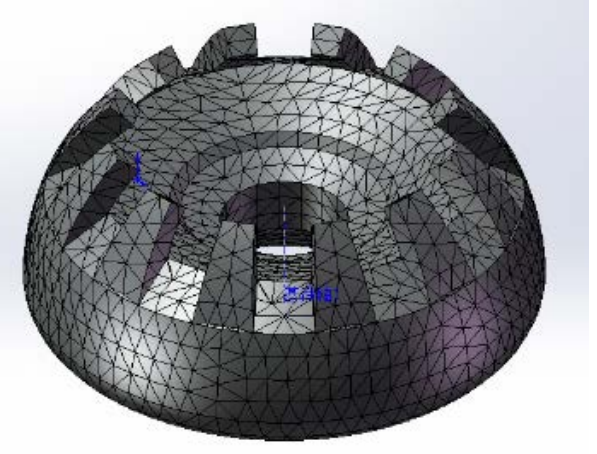

Fig. 6 The finite-element model of rubber core

Table 2 material parameters rubber core

\begin{tabular}{cc}
\hline Parameter term & Rubber \\
\hline Elastic modulus/MPa & 6.1 \\
Poisson ratio & 0.49 \\
Yield strength/MPa & 835 \\
Tensile strength/MPa & 980 \\
Mass density/(kg $\bullet \mathrm{m}-3)$ & 7870 \\
Shear modulus/MPa & 82400 \\
\hline
\end{tabular}

Imposing constraints on the bottom and outer side of the model of rubber core, carrying uniform load of 21MPa on the area of claw and upper torus, and then calculating.

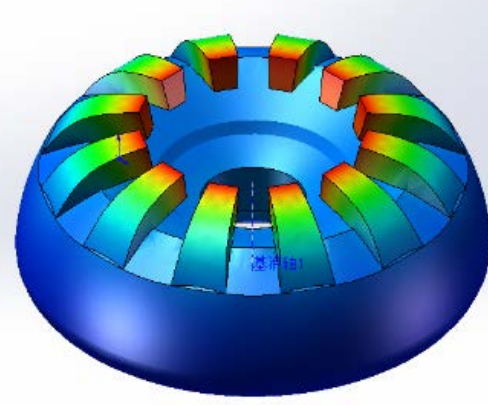

Fig. 7 The displacement nephogram of rubber core

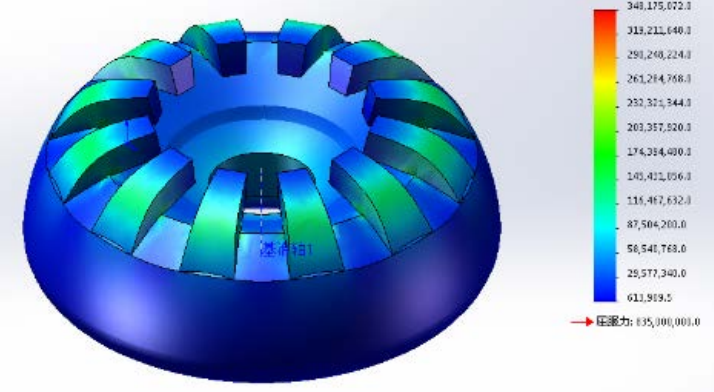

Fig.8 The stress nephogram of rubber core

According to the calculation result, the top of rubber core is biggest, the relative displacement is $0.1503 \mathrm{~mm}$, as shown in Figure 7. The maximum Von Mises pressure is 348MPa, it is less than yield strength, as shown in Figure 8. The above shows that the shell meets the requirements.

\section{Conclusions}

(1)Carrying finite-element analysis on the shell and rubber core of spherical BOP, the displacement and stress nephogram of shell and rubber core and the region of stress concentration could be obtained.The region of stress concentration is the junction of ball cover and base, and the rubber core is on its top.

(2)The results of the finite-element analysis has a guiding significance on the analysis of bearing capacity,design,detection and safety evaluation of spherical BOP. 
(3)Taking the three-dimensional software SolidWorks as a platform, spherical BOP 3D model is established, carrying force simulation on crucial component of spherical BOP, the closest real distributed law of pressure and deformation was obtained. If we combine experiment reasonably, Design cost could be greatly reduced and the design period could be shortened.

\section{References}

[1] Fu Hailong, Wang Jinyou, Jia Guangzheng, et al. Finite element analysis of the key components of the ram blowout preventer[J]. China petroleum machinery, 2008, 36(08): 25-27.

[2]Chang Yulian, Wei Jing, Gao Sheng, et al. Technical development of independent non pressure well operation equipment[J]. Oil Field Equipment, 2011, 40(4): 12-16.

[3]Sizeer P. Appratus for moving a well flow conductor into or out of a well[P]. U.S.: Patent No.3215203.

[4]Lin Jun. Mechanical analysis of the rubber skeleton of spherical BOP[J]. China petroleum machinery, 1990(6): 1-5. 